\title{
Integrated genomic-metabolic classification of acute myeloid leukemia defines a subgroup with NPM1 and cohesin/DNA damage mutations
}

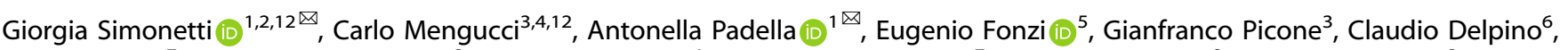 \\ Jacopo Nanni ${ }^{7}$, Rossella De Tommaso ${ }^{2}$, Eugenia Franchini ${ }^{1}$, Cristina Papayannidis ${ }^{7}$, Giovanni Marconi ${ }^{8}$, Martina Pazzaglia ${ }^{2}$, \\ Margherita Perricone ${ }^{2}$, Emanuela Scarpi ${ }^{5}{ }^{5}$, Maria Chiara Fontana ${ }^{1}$, Samantha Bruno ${ }^{2}$, Michela Tebaldi ${ }^{5}$, Anna Ferrari (D) ${ }^{1}$, \\ Maria Teresa Bochicchio ${ }^{1}$, Andrea Ghelli Luserna Di Rorà ${ }^{1}$, Martina Ghetti ${ }^{1}$, Roberta Napolitano ${ }^{1}$, Annalisa Astolfi ${ }^{9}$, Carmen Baldazzi ${ }^{7}$, \\ Viviana Guadagnuolo ${ }^{2}$, Emanuela Ottaviani ${ }^{7}$, Ilaria lacobucci iD ${ }^{10}$, Michele Cavo ${ }^{2,7}$, Gastone Castellani ${ }^{2}$, Torsten Haferlach ${ }^{11}$, \\ Daniel Remondini ${ }^{4}{ }^{4}$, Francesco Capozzi (D) $^{3}$ and Giovanni Martinelli ${ }^{1}$
}

(c) The Author(s) 2021

\begin{abstract}
Although targeting of cell metabolism is a promising therapeutic strategy in acute myeloid leukemia (AML), metabolic dependencies are largely unexplored. We aimed to classify AML patients based on their metabolic landscape and map connections between metabolic and genomic profiles. Combined serum and urine metabolomics improved AML characterization compared with individual biofluid analysis. At intracellular level, AML displayed dysregulated amino acid, nucleotide, lipid, and bioenergetic metabolism. The integration of intracellular and biofluid metabolomics provided a map of alterations in the metabolism of polyamine, purine, keton bodies and polyunsaturated fatty acids and tricarboxylic acid cycle. The intracellular metabolome distinguished three AML clusters, correlating with distinct genomic profiles: NPM1-mutated(mut), chromatin/spliceosome-mut and TP53-mut/aneuploid AML that were confirmed by biofluid analysis. Interestingly, integrated genomic-metabolic profiles defined two subgroups of NPM1-mut AML. One was enriched for mutations in cohesin/DNA damage-related genes (NPM1/cohesin-mut $\mathrm{AML}$ ) and showed increased serum choline + trimethylamine-N-oxide and leucine, higher mutation load, transcriptomic signatures of reduced inflammatory status and better ex-vivo response to EGFR and MET inhibition. The transcriptional differences of enzymeencoding genes between NPM1/cohesin-mut and NPM1-mut allowed in silico modeling of intracellular metabolic perturbations. This approach predicted alterations in NAD and purine metabolism in NPM1/cohesin-mut AML that suggest potential vulnerabilities, worthy of being therapeutically explored.
\end{abstract}

Leukemia (2021) 35:2813-2826; https://doi.org/10.1038/s41375-021-01318-x

\section{INTRODUCTION}

Current personalized therapeutic approaches in acute myeloid leukemia (AML) are generally restricted to those patients with identifiable and targeteable genomic lesions [1-3]. However, these approaches do not target interactions between cancer-related features and homeostatic mechanisms that define the leukemic phenotype.

The metabolome is the result of genome- and proteomewide interactions and is shaped by microenvironmental factors.
The biofluid metabolome has been extensively investigated to identify predictive signatures in cardiovascular disorders [4], diverticular disease [5] and diabetes [6], and specific metabolic profiles have been associated with cancer risk [7]. In oncology, metabolomics is a valuable approach for diagnosis, prognostication, and disease monitoring [8]. A paradigmatic example in AML is the accumulation of serum, urine, and intracellular 2-hydroxyglutarate (2-HG) in IDH1/2-mutated (mut) cases $[9,10] .2-\mathrm{HG}$ is an oncometabolite [11, 12], predicts

\footnotetext{
'Biosciences Laboratory, IRCCS Istituto Romagnolo per lo Studio dei Tumori (IRST) "Dino Amadori", Meldola, FC, Italy. ${ }^{2}$ Department of Experimental, Diagnostic and Specialty Medicine, University of Bologna, Bologna, Italy. ${ }^{3}$ Department of Agricultural and Food Sciences, University of Bologna, Cesena, FC, Italy. ${ }^{4}$ Department of Physics and Astronomy, University of Bologna, Bologna, Italy. ${ }^{5}$ Unit of Biostatistics and Clinical Trials, IRCCS Istituto Romagnolo per lo Studio dei Tumori (IRST) "Dino Amadori", Meldola, FC, Italy. ${ }^{6}$ Departamento de Ingeniería Química, Universidad Nacional del Sur, Bahía Blanca, Argentina. ${ }^{7}$ IRCCS Azienda Ospedaliero-Universitaria di Bologna, Istituto di Ematologia "Seràgnoli", Bologna, Italy. ${ }^{8}$ Hematology Unit, IRCCS Istituto Romagnolo per lo Studio dei Tumori (IRST) "Dino Amadori", Meldola, FC, Italy. ${ }^{9}$ Giorgio Prodi" Cancer Research Center, University of Bologna, Bologna and Department of Biomedical and Specialty Surgical Sciences, University of Ferrara, Ferrara, Italy. ${ }^{10}$ Department of Pathology, St. Jude Children's Research Hospital, Memphis, TN, USA. ${ }^{11}$ MLL Munich Leukemia Laboratory, Munich, Germany. ${ }^{12}$ These authors contributed equally: Giorgia Simonetti, Carlo Mengucci. email: giorgia.simonetti@irst.emr.it; antonella.padella@irst.emr.it
} 
clinical outcome [13], and is a noninvasive biomarker of disease activity [10].

Aberrant enzymatic activity drives cancer metabolic reprogramming and cooperates with mutations of tumor suppressors and oncogenes in pathogenesis. For example, AML cells reduce both host insulin sensitivity and secretion to increase glucose availability for malignant cells [14]. The glycolytic pathway sustains leukemia maintenance and progression. AML cells have a higher mitochondrial $(\mathrm{mt})$ mass and oxygen consumption rate than normal hematopoietic cells [15]. Moreover, leukemia stem cells (LSCs) are addicted to oxidative phosphorylation (OXPHOS) for energy production [16]. OXPHOS is sustained by elevated amino acid metabolism in LSC from de novo AML [17], with cysteine playing a crucial role [18], and is controlled by glutamine levels [19]. Targeted inhibition of these pathways, among others, induces cell death and/or differentiation of AML cells $[15,17-$ 21]. However, the specific response of AML molecular subtypes to agents targeting metabolism has been rarely investigated [22-25].

Here we report an integrated genomic-metabolic study in AML that identified, based on intracellular and the biofluid metabolic profile, a specific NPM1-mut AML subgroup characterized by mutations of genes involved in DNA damage response and/or chromatid cohesion (NPM1/cohesin-mut) and high levels of serum choline + trimethylamine-N-oxide, and leucine. In silico modeling of the intracellular metabolome based on transcriptomic data highlighted perturbations in the purine and NAD metabolic pathways as NPM1/cohesin-mut-specific alterations.

\section{MATERIALS/SUBJECTS AND METHODS Metabolomic study design}

Participants were included if they were free from infective, autoimmune, celiac, or metabolic diseases such as diabetes and dyslipidaemia. Kidney and liver integrity were also checked. Subjects with acute or chronic renal or hepatic disease, renal or hepatic impairment, cardiovascular disease or a history of neoplasia were excluded from the control cohort. Serum samples from of $119 \mathrm{AML}$ and 145 healthy subjects and urine samples of $103 \mathrm{AML}$ and 139 controls were collected in the fasting state (in the morning). All participants were Caucasian except for 5 (3.4\%) healthy controls and six (5\%) AML patients. To reduce potential bias and variation unrelated to AML pathogenesis and to ensure that the observed metabolic differences were not due to external confounders, we collected, when possible, two independent serum and urine samples from each patient (more than $50 \%$ of cases). Moreover, information on age, gender (the cohorts were balanced for gender), race, health status, diet, drug intake, physical exercise was collected along with specimens and used to filter nuclear magnetic resonance (NMR) spectra during the quality control procedures.

\section{Nuclear magnetic resonance (NMR) spectroscopy}

Serum and urine samples were analyzed by NMR spectroscopy (Supplementary Methods). A stochastic GridSearch was implemented to select the best combination of parameters for dimensionality reduction and classifier performances. Unsupervised and supervised dimensionality reduction were performed using principal component analysis (PCA) and partial least squares discriminant analysis (PLSDA)-sparse(s)PLSDA, respectively. For subset extraction, weights were obtained after signal smoothing via signal-to-noise ratio threshold (which was essential due to unavoidable use of data scalers for dimensionality reduction). The latent components of spectra containing maximum information related to molecular features were identified by a genomic-guided semisupervised approach. This means that the combination of urine and sera latent components used for clustering is extracted with classifiers-derived scores, from classifiers trained with the purpose of discriminating TP53-mut/aneuploid, NPM1-mut and chromatin/spliceosome-mut samples. Signals in the spectra corresponding to loadings and weights emerging from different tasks were checked for alignment. To minimize the possibility of confounding effects, every step of each classification and clustering task was crossvalidated through suitable k-folds, stratified for gender and age when possible depending on class sizes and sample sizes for the tasks.

\section{Mass spectrometry (MS)-based metabolomics and data analysis}

MS-based meabolomics was performed using an ultraperformance liquid chromatography (Waters ACQUITY, Waters, Milford, MA, USA) and a Q-Exactive high resolution/accurate mass spectrometer (Thermo Fisher Scientific, Waltham, MA, USA) interfaced with a heated electrospray ionization (HESI-II) source and Orbitrap mass analyzer operated at 35.000 mass resolution (Metabolon, Morrisville, NC, USA). Raw data were extracted, peakidentified and QC processed. Compounds were identified by comparison with library entries of purified standards or recurrent unknown entities. Peaks were quantified using area-under-thecurve. Metabolite levels were normalized to DNA content.

\section{Whole exome sequencing (WES)}

WES was performed on $100 \mathrm{AML}$ cases, 17 belonging to a published dataset [26] and 83 new cases. Libraries were prepared from matched tumor and germline DNA (saliva or complete remission samples, Nextera Rapid Capture Expanded and TruSeq Rapid Exome kits, Illumina, San Diego, CA, USA) according to manufacturer's protocol, and 75/125-bp paired-end sequences were generated (Illumina NextSeq550/HiSeq2500, Illumina). A detailed description is reported in the Supplementary Methods and Tables S1, S2. Sequencing data are available in the European Genome-Phenome Archive (EGAS00001005422).

\section{Constraint-based metabolic network analysis}

We translated gene expression alterations into constraints reducing the feasible space of a metabolic network model (adapted from Shlomi et al. [27]). The impact of a set of these constraints on the feasible space of the metabolic network was evaluated by calculating the minimum and maximum reaction rates (flux variability analysis, FVA), and the instantaneous capability of the network to produce/consume a certain metabolite. Details are reported in the Supplementary Methods. Codes used in constraint-based metabolic network analysis are available in https://github.com/cladelpino/GenePerturbations.

\section{Statistics}

Associations in contingency tables were performed by the Monte Carlo $(B=1000,000)$ simulated Fisher's exact test. Continuous variables were compared with Mann-Whitney, Kolmogorov-Smirnov, Kruskal-Wallis test, or Welch's $t$-test. All tests were performed using either python v3.6.5 [28] (packages scipy v1.3.2 [29], statsmodels v0.10.1 [30]) or Rv3.6.3 [31]. When appropriate, $p$ values were adjusted for multiple comparisons using the Bonferroni or Benjamini-Hochberg method. To investigate the distribution of sera profile according to blast percentage, samples were divided in three classes (bone marrow: $20-49 \%, 50-74 \%, \geq 75 \%$ blasts, peripheral blood: $<30 \%, 30-69 \%$, $\geq 70 \%$ blasts, according to tertiles). In the drug response analysis, the average area-under-the curve values of the two cohorts were compared. NMR peaks, signal integrals (related to metabolite concentration) and intracellular metabolite levels among three groups were compared by Kruskal-Wallis test. For intracellular metabolite levels Welch $t$-test was also used as post-hoc test. Random Forest analysis was used to estimate the accuracy of individual classification in each group based on metabolomic data. Metabolic pathway analysis was performed using Metaboanalyst (http://www.metaboanalyst.ca) with KEGG annotation. A threshold 

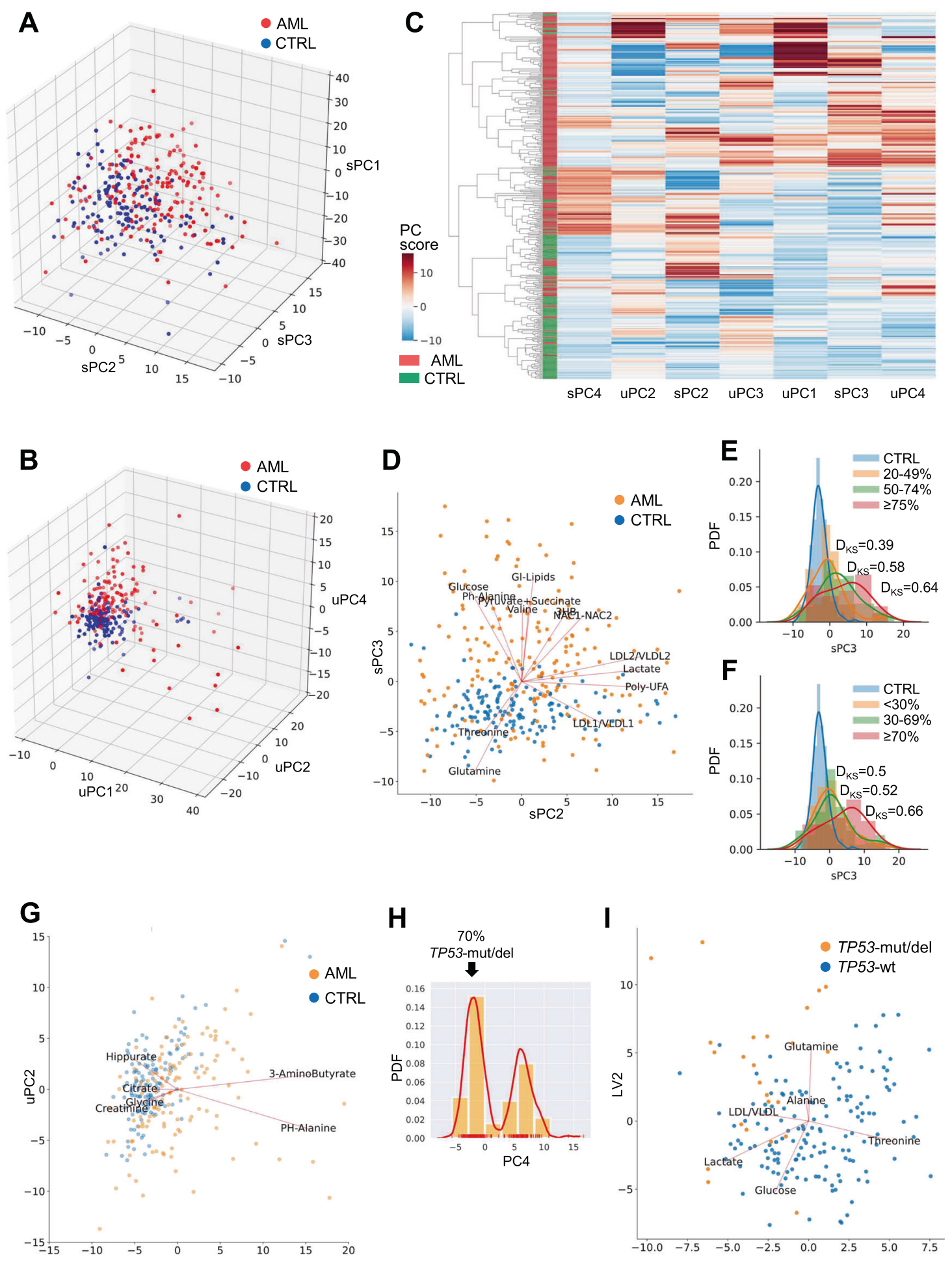

of five standard deviations from the mean of the control population was used for the identification of outliers.

\section{RESULTS}

The combined analysis of serum and urine profiles improves AML metabolic characterization

Given that the metabolite composition of biofluids reflects the real-time activity of all biochemical processes in the body and that leukemic cells alter systemic physiology [14], we compared the profile of blood (Table S3) and urine (Table S4) metabolites of AML patients (serum: 88 at diagnosis and 31 at relapse, urine: 80 at diagnosis and 23 at relapse) and healthy controls (CTRL, serum: 145, urine: 139).

The metabolomic profile provided efficient discrimination between patient and CTRL both at serum and urine level, with an accuracy of $83 \%$ (Figs. $1 \mathrm{~A}$ and S1) and $85 \%$ (Figs. 1B and S2), respectively. Since patient and CTRL cohorts were not age- 
Fig. 1 Serum and urine metabolic profile of AML. A 3D representation of principal component (PC)1, PC2, and PC3 projection of serum NMR data of AML and healthy controls (CTRL), which accounted for 53\% of the total explained variance. B 3D representation of PC1, PC2, and PC4 projections of urine NMR data of AML and healthy controls. C Hierachical clustering of AML and controls using integrated serum ( $n=3)$ and urine $(n=4)$ PCs, selected as the best combination of predictive features by comparing an AdaBoost Classifier and a SVM Classifier. The integration yielded an enhanced coherence in adjacency between AML and controls compared with single biofluid analysis. Each component contains linear combinations of signature metabolites shown in biplots for both sera and urine samples. Colors indicate the score on each PC. D BiPlot on PCA reduced space of serum NMR data. Metabolites showing significant alterations $(p<0.05)$ were plotted along their maximum variance direction in the PCA score space. Only completely template-matched signals were reported. E Estimated probability density functions (PDFs) of serum PC3 scores of AML cases according to bone marrow blast percentage (20-49\%: $p=1.66 \mathrm{e}-04 ; 50-74 \%: p=6.47 \mathrm{e}-10 ; \geq 75 \%: p$ $=1.67 \mathrm{e}-15)$ and $\mathbf{F}$ peripheral blood blast percentage $(<30 \%: p=8.85 \mathrm{e}-08 ; 30-69 \%: p=5.98 \mathrm{e}-10 ; \geq 70 \%: p=8.33 \mathrm{e}-15)$. The similarity between each AML blast count class and CTRL was computed using the score distribution of serum PC3, which is the latent variable best separating $A M L$ and CTRL in the metabolic latent space $\left(D_{K S}\right.$ : absolute value of the maximal difference between the cumulative function of two distributions, representing the maximal distance between them, according to Kolmogorov-Smirnov statistics). G BiPlot on PCA reduced space of urine NMR data. Metabolites were plotted as in (D). H Serum PC4 scores in AML patients (median value: group 1, -1.94 and group 2, 6.35). I BiPlot on PLSDA reduced space (from a 5-PLSDA-component AdaBoost classification) for TP53-wt and TP53-mut/del AML. Metabolites were plotted along their maximum variance direction in the PLSDA score space (LV latent variables).

Table 1. Metabolic alterations in serum and urine samples from AML patients compared with those from controls.

\begin{tabular}{|c|c|c|c|c|}
\hline Metabolite & Biplot name & Changes in AML vs. CNTRL & Biofluid & $\begin{array}{l}\text { Kruskal-Wallis } \\
p \text {-value }\end{array}$ \\
\hline Glycerol of lipids & Gl-Lipids & $\uparrow$ & Serum & $<0.001$ \\
\hline Glutamine & Glutamine & $\downarrow$ & Serum & $<0.001$ \\
\hline Lactate & Lactate & $\uparrow$ & Serum & 0.033 \\
\hline $\mathrm{N}$-acetylglycoproteins (1 and 2) & NAC1/NAC2 & $\uparrow$ & Serum & $<0.001$ \\
\hline Polyunsaturated fatty acids & Poly-UFA & $\uparrow$ & Serum & 0.008 \\
\hline Pyruvate + Succinate & Pyruvate + Succinate & $\uparrow$ & Serum & 0.033 \\
\hline Threonine & Threonine & $\downarrow$ & Serum & $<0.001$ \\
\hline Valine & Valine & $\uparrow$ & Serum & 0.022 \\
\hline Creatinine & Creatinine & $\downarrow$ & Urine & $<0.001$ \\
\hline Glycine & Glycine & $\downarrow$ & Urine & 0.007 \\
\hline Hippurate & Hippurate & $\downarrow$ & Urine & $<0.001$ \\
\hline
\end{tabular}

$\uparrow$ : up; $\downarrow$ : down.

matched (median age: AML, 67-years (18-90), CTRL, 57-years (23-75)), we verified that age had no significant effects on the classification (Table S5). Notably, the integration of serum and urine data yielded an average accuracy of $90 \%$ in the separation of AML and CTRL (Fig. 1C), by using a reduced number of features (Fig. S3) compared with the analysis of each biofluid per se. In serum, PC2-3 space gave the best 2D combination for AML-CTRL separation, with 13 metabolites showing signficantly different levels $(p<0.05$, Fig. 1D and Table 1). These metabolites were not significantly correlated with age or gender (Table S6). Amino acid and tricarboxylic acid cycle (TCA) cycle byproducts, that had increased concentration in AML except for glutamine and threonine, mainly represented variance in $\mathrm{PC} 3$, while lactate and fatty acid metabolism compounds accounted for variance in PC2 (Fig. 1D). When looking at sample distribution along serum PC3, that provided a good discrimination between $A M L$ and CTRL, we observed that all AML subgroups were significantly different from normal cases, independently of bone marrow or peripheral blast percentage (Fig. 1E, F). Moreover, a low bone marrow blast percentage $(20-49 \%$, Fig. $1 \mathrm{E})$ and a high peripheral blood blast percentage $(\geq 75 \%$, Fig. $1 \mathrm{~F})$ resulted in a reduced and increased distance from $C T R L$, respectively.

Moreover, we detected increased concentration of 3aminobutyrate and phenylalanine in the urine of AML patients compared with CTRL (Fig. 1G, Tables 1 and S6). Citrate, creatinine, and hippurate, which are among the most abundant urine components, showed low levels in AML, suggesting reduced excretion. Similarly, decreased glycine was indicative of reduced catabolism.

Notably, two groups of patients were distinguished by serum metabolites in PC4 $(p<0.001)$, and one of them included $70 \%$ of TP53-mut/deleted(del) AML (Fig. 1H). When comparing TP53-mut/ del and wild-type (wt) AML, we found lower levels of threonine and glucose in TP53-mut/del cases (Fig. 11), that suggested an increased cellular uptake, likely aimed at satisfying macromolecule biosynthesis and bioenergetic requirements [32], with reduced lactate excretion [33].

Overall, integration of serum and urine metabolomics improved the prediction accuracy with respect to single biofluid classification. 
$\mathrm{CD}^{+} 4^{+}$and $\mathrm{CD} 33^{+} \mathrm{AML}$ cells have dysregulated lipid, amino acid, nucleotide, and bioenergetic metabolism

To obtain a metabolic fingerprint of AML, we performed intracellular metabolic profiling of leukemic cells $\left(35 \mathrm{CD}_{3} 4^{+}\right.$and $15 \mathrm{CD}^{\mathrm{C}} 3^{+}$ isolated bone marrow (BM) blasts) and compared them with 21 normal cord blood (CB) $\mathrm{CD}_{3}{ }^{+}$and 21 normal $\mathrm{CD}_{3} 3^{+}$peripheral blood (PB) samples from healthy subjects. $C D 34^{+} \mathrm{AML}$ and $\mathrm{CD} 33^{+}$ $A M L$ segregated from their normal counterparts (Fig. 2A, B), with a
A

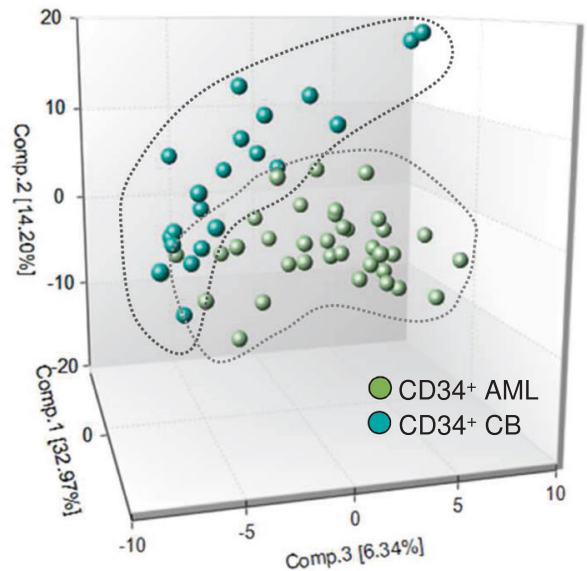

C

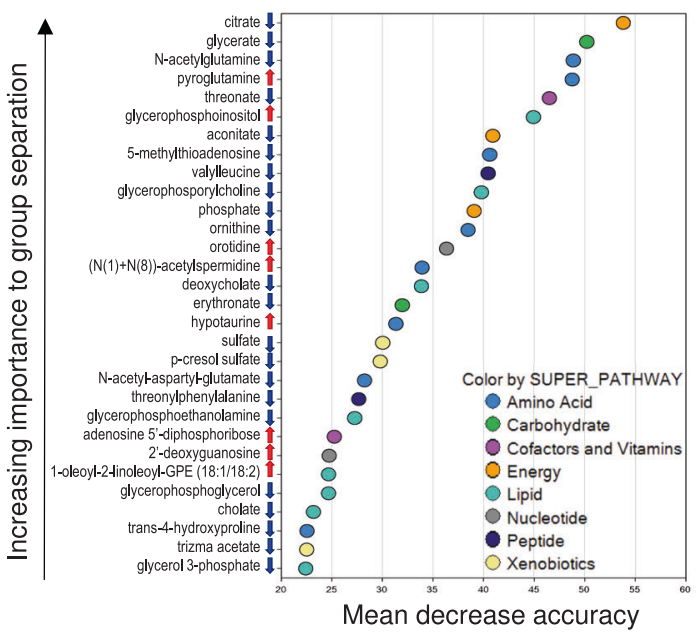

D

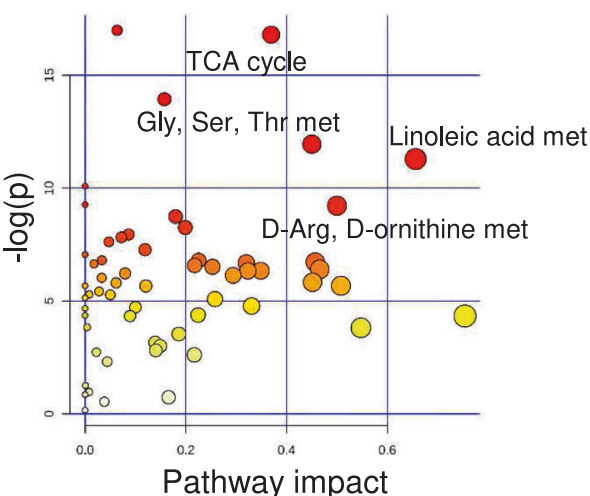

B

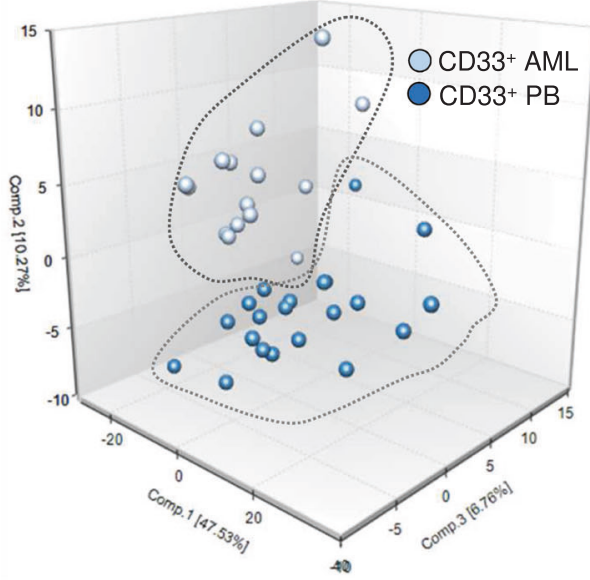

$\mathbf{E}$

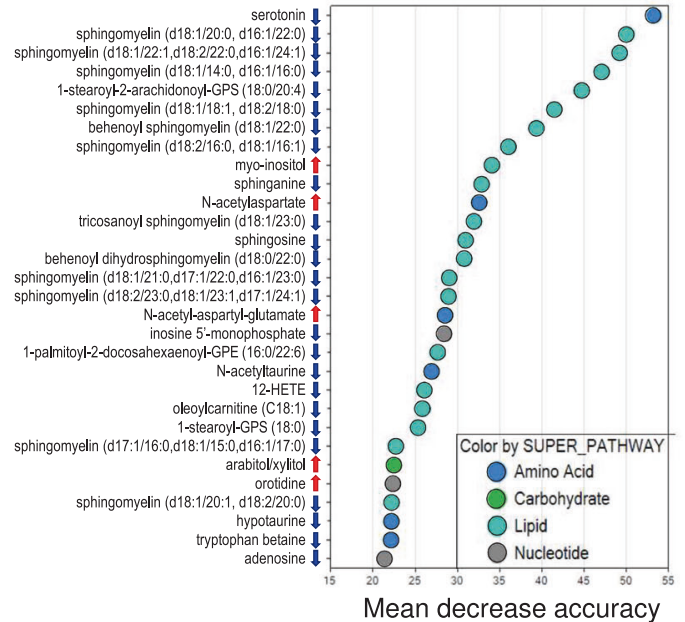

$\mathbf{F}$

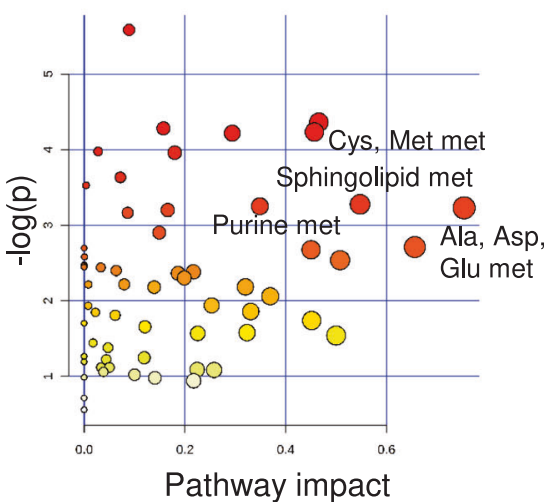

Fig. 2 Intracellular metabolomics of AML. PCA of the metabolic profile of $\mathbf{A} C D 34^{+}$and $\mathbf{B} C D 33^{+}$AML cells compared to their healthy control populations (CD34 ${ }^{+} \mathrm{CB}$ and $\mathrm{CD} 33^{+} \mathrm{PB}$ cells). C Biochemical importance plot of the top 30 metabolites contributing to group separation between $\mathrm{CD} 34^{+} \mathrm{AML}$ and $\mathrm{CD} 34^{+} \mathrm{CB}$ stem-progenitor cells. Red and blue arrows indicate increased or decreased metabolite levels in AML cells compared with CTRL cells (|fold change $\mid \geq 2, q \leq 0.05$ ), respectively. D Altered metabolic pathways in CD34 ${ }^{+}$AML cells. The most significant pathways with the strongest impact on $\mathrm{CD} 34^{+} \mathrm{AML}$ cells are shown. E Biochemical importance plot of the top 30 metabolites contributing to group separation between CD33 $3^{+} \mathrm{AML}$ blasts and $\mathrm{CD} 33^{+} \mathrm{PB}$ cells from CTRL (red and blue arrows as in (C)). $\mathbf{F}$ Altered metabolic pathways in $\mathrm{CD}^{+} 3^{+} \mathrm{AML}$ cells. The most significant pathways with the strongest impact on $\mathrm{CD} 33^{+} \mathrm{AML}$ cells are shown. 
predictive accuracy of $85.7 \%$ and $94.4 \%$, respectively (Fig. S4A, B), but not from each other (Fig. S4C). Among the 300 detected metabolites, 66 and 35 were down- and upregulated, respectively, in $\mathrm{CD}^{+}{ }^{+} \mathrm{AML}$ cells, while 102 and 19 showed reduced and increased levels, respectively, in $\mathrm{CD}_{3} 3^{+} \mathrm{AML}$ compared with their control group. No significant differences in metabolite levels were detected between $\mathrm{CD}_{3} 4^{+}$and $\mathrm{CD}_{3}{ }^{+} \mathrm{AML}$.

The top scored 30 biochemicals that distinguished $\mathrm{CD} 34^{+} \mathrm{AML}$ from $\mathrm{CD} 34^{+} \mathrm{CB}$ cells were primarily involved in bioenergetics, amino acid, and lipid metabolism (Fig. 2C). Overall, 41 pathways were dysregulated in $\mathrm{CD} 34^{+} \mathrm{AML}$, with TCA cycle, D-Arginine and D-ornithine and linoleic acid metabolism showing the strongest impact (Fig. 2D and Table S7).

When comparing $\mathrm{CD}_{3} 3^{+} \mathrm{AML}$ and $\mathrm{CD}_{3}{ }^{+} \mathrm{PB}$, the top discriminating 30 biochemicals included lipids, nucleotides, and amino acid metabolism (Fig. 2E), with alanine, aspartate and glutamate, cysteine and methionine, purine and sphingolipid metabolic pathways showing the strongest impact (Fig. $2 \mathrm{~F}$ and Table S8).

Lipid, amino acid, nucleotide, and bioenergetic metabolism were confirmed as the most widely altered pathways when comparing the whole AML and CTRL cohorts (Fig. S4D and Table S9; 17 increased and 147 decreased metabolites), which were separated with a predictive accuracy of $89.1 \%$ (Fig. S4E).

\section{Integrated intracellular and biofluid metabolomics highlighted alterations in the metabolism of polyamine, purine, keton bodies and polyunsaturated fatty acids and in the TCA cycle in AML}

After showing a distinct metabolomic profile for leukemic compared to normal $\mathrm{CD}_{3} 4^{+}$or $\mathrm{CD}_{3} 3^{+}$cells, we next focused on the significantly dysregulated metabolic pathways. We observed decreased arginine, methionine, and proline in leukemic cells, that suggested elevated polyamine biosynthesis (S-adenosylmethionine, 5-methylthioadenosine, and N1-acetylspermidine in $\mathrm{CD} 33^{+}$and $\mathrm{CD} 34^{+}$cells, respectively, Fig. $3 \mathrm{~A}$ ), which in turn supports cell proliferation [34]. Accordingly, the low levels of purine nucleotides (Fig. 3B) may indicate enhanced production of adenosine $5^{\prime}$-triphosphate and guanosine 5'-triphosphate that are crucial for providing cellular energy and intracellular signaling, respectively [35]. Tumor growth was also supported by elevated $\mathrm{N}$-acetylaspartate levels in leukemic cells (Fig. 4A) [36]. Of note, in the CD33 ${ }^{+}$cohort, NPM1-mut AML scored as outliers for their high levels of the $\mathrm{N}$-acetylaspartate derivative $\mathrm{N}$ acetyl-aspartyl-glutamate $(90.9 \%$ and $25.0 \%$ of NPM1-mut AML among outliers and non-outliers, respectively, $p=0.033$ ). Moreover, high levels of reduced glutathione (Fig. $3 \mathrm{~A}$ ) and ophtalmate (data not shown) were indicative of elevated cellular oxidative stress, and increased 3-hydroxybutyrate in the serum of patients and of 3-hydroxybutyrylcarnitine in leukemic cells reflected heightened ketogenesis in AML (Fig. 4A and Table S10). Polyunsaturated fatty acids (Fig. 4B) and glucose (Fig. 4A) were elevated in the serum of patients but reduced in $\mathrm{CD}_{3}{ }^{+}$and/or $\mathrm{CD} 34^{+}$leukemic cells compared with normal ones, suggesting the need for a constant energy reservoir that is rapidly consumed by cells. The reduced levels of intracellular TCA intermediates and of serum glutamine were also indicative of increased bioenergetics requirement, especially in the CD34 compartment (Fig. 4A). This requirement was further supported by decreased levels of amino acid sources of pyruvate (e.g. threonine, glycine, serine, alanine), with a significant increase of serum lactate, an endproduct of glycolysis and glutaminolysis (Fig. 4A). In parallel, intracellular lactate levels were lower in both $\mathrm{CD}_{3} 4^{+}$and $\mathrm{CD}_{3} 3^{+}$ AML than normal cells, thus suggesting a high excretion capacity (Table S10).

\section{Metabolic clusters define AML subgroups with different genomic features}

We then classified AML cases according to their intracellular metabolic profile. Unsupervised hierarchical clustering clearly defined 3 clusters (Fig. 5A and Table S11). The top 15 metabolites that better distinguished the 3 clusters included amino acids and their derivatives (e.g. tyrosine, phenylalanine, tryptophan, threonine, lysine), intermediates of purine and pyrimidine metabolism (e.g. hypoxanthine, adenosine $5^{\prime}$-monophosphate, uridine) and lipids (e.g. palmitoyl sphingomyelin, cholesterol), that showed high, intermediate and low levels in cluster 1,2 and 3, respectively (Fig. 5B). In order to integrate genomics (Table S12) and metabolomics, we assigned each sample to a molecular class [3]. Cluster-1 was enriched for NPM1-mut AML (50.0\%), cluster-2 for cases with altered chromatin/spliceosome genes (37.5\%), and cluster-3 for TP53-mut/aneuploid AML (34.4\%, $p=0.023$, Fig. 5C). We then investigated differences at serum and urine level across genetic categories (chromatin/spliceosome-mut, NPM1-mut, TP53mut/aneuploid AML, $n=71$ ) and identified 4 NMR clusters (Fig. 5D). Genomic categories associated with specific biofluid metabolic cluster (clusters 2, 3, and 4, $p=0.040$, Fig. 5E), in accordance with the intracellular metabolic profiles.

High levels of serum tyrosine, threonine, and citrate correlated with the cluster enriched for chromatin/spliceosome-mut. Viceversa, low levels of these metabolites were detected in the cluster enriched for NPM1-mut (Fig. 5F). The cluster associated with TP53-mut/aneuploid AML displayed intermediate threonine and tyrosine levels and high citrate in the serum compared to the other two clusters. Notably, tyrosine and threonine showed high intracellular levels in the NPM1-mut enriched cluster compared with the other clusters (mean decrease accuracy $=0.010$ and 0.005 , respectively, Fig. 5B), suggesting an increased intracellular need and/or uptake leading to serum depletion.

\section{NMR-driven metabolic classification identifies two subgroups of NPM1-mut patients}

Our data so far described a significant association between genomic and metabolic profiles. However, even within the same genomic category, different subgroups can be identified according to combinatorial mutation patterns and consequently they may show metabolic differences. This hypothesis was confirmed in patients carrying NPM1 mutations, in whom the metabolic profiles defined two distinct subgroups. NPM1-mut patients with higher serum levels of choline + trimethylamine $\mathrm{N}$-oxide, leucine and leucine + lysine ( $p<0.05$, Fig. 6A) were enriched for co-occurring mutations in cohesin complex and DNA damage genes (SMC1A, SMC3, RAD21, STAG2, ATM, ATR, BRCA2, named NPM1/cohesinmut), compared with NPM1-mut patients from the other metabolic group $(60.0 \%$ versus $9.1 \%$ of cases, respectively, $p=0.024)$.

In order to gain insights into molecular mechanisms associated with the metabolic differences between NPM1/ cohesion-mut and NPM1-mut AML, we analyzed paired exome and transcriptome (Fig. S5) data from the TCGA and BEAT AML datasets for the same genetic subgroups. Twenty-three percent of NPM1-mut AML (32/137) also carried at least one alteration in recurrently mutated genes [1] belonging to the cohesin complex or DNA damage pathways. Compared with NPM1-mut AML, $N P M 1 /$ cohesin-mut cases were characterized by a lower white blood cell count (39.7 vs. 64.1 cells $/ \mathrm{mm}^{3}, p=0.006$ ) and a significantly higher mutation load (average mutation number: 15 vs. 9, $p<0.001$ ), with lower frequency of IDH1-2/TET2 mutations $(21.9 \%$ vs. $46.7 \%$ of NPM1-mut, $p=0.014$, Fig. $6 \mathrm{~B})$. FLT3 alterations were evenly distributed between the two groups (Fig. 6B) and no differences were observed in clinical outcome (Fig. S6A). 

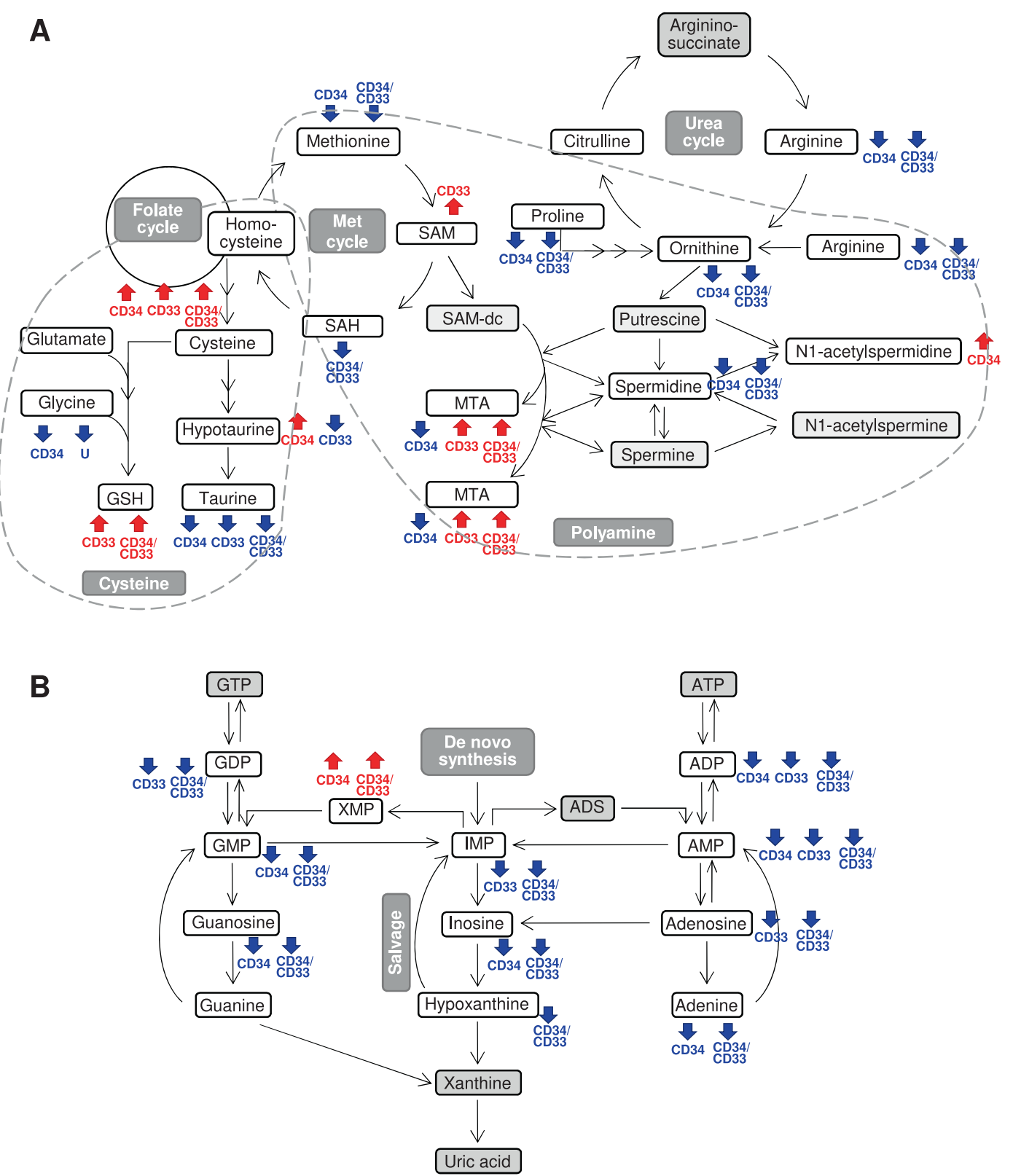

Fig. 3 Schematic representation of polyamine, cysteine, and purine metabolic pathways integrating intracellular and biofluid metabolomic data. A Polyamine and cysteine metabolic pathway and urea cycle. B Purine metabolism. Red and blue arrows/text indicate increased or decreased metabolite levels in AML cells compared with their CTRL, respectively (|fold change $\mid \geq 2, q \leq 0.05)$ and in the urine of patients compared with CTRL $(p<0.05)$. Gray metabolite boxes indicate the ones that were not detected by MS analysis. The schemes report the most relevant metabolites in the pathway according to metabolomic data (ADP adenosine $5^{\prime}$-diphosphate, ADS adenosine, AMP adenosine 5'-monophosphate, ATP adenosine 5'-triphosphate, dcSAM decarboxylated S-adenosylmethionine, GDP guanosine 5'-diphosphate, GMP guanosine 5'-monophosphate, GTP guanosine 5'-triphosphate, GSH reduced glutathione, IMP inosine 5'-monophosphate, MET methionine, MTA 5-methylthioadenosine, SAH S-adenosylhomocysteine, SAM S-adenosylmethionine, XMP xanthosine $5^{\prime}$-monophosphate).

At transcriptional level, signatures of cellular response to cytokines and JAK-STAT cascade were significantly downregulated in NPM1/cohesin-mut AML (Fig. 6C). Accordingly, NPM1/cohesinmut AML showed reduced expression of genes involved in the regulation of immune and inflammatory response, along with others related to cell differentiation and metabolism (Fig. 6D).

We then compared the ex vivo response of NPM1/cohesin-mut and NPM1-mut AML to a panel of targeted agents $(n=122$, BEAT AML [1]). NPM1/cohesin-mut AML showed decreased sensitivity to the Aurora kinase A inhibitor MLN8054 and the FLT3/JAK inhibitor
Midostaurin but responded better to SYK, MET, and EGFR inhibitors (Entospletinib, JNJ-38877605, Crizotinib, Foretinib, Lapatinib, Pelitinib, Fig. 6E).

These data suggest that the co-occurrence of different mutations with altered NPM1 may confer a distinct metabolic, transcriptomic, and drug sensitivity profile to the leukemic cells.

Predicting metabolic specificities of NPM1/cohesin-mut AML Seven downregulated genes in NPM1/cohesin-mut compared with NPM1-mut AML encoded for enzymes involved in nucleotide 


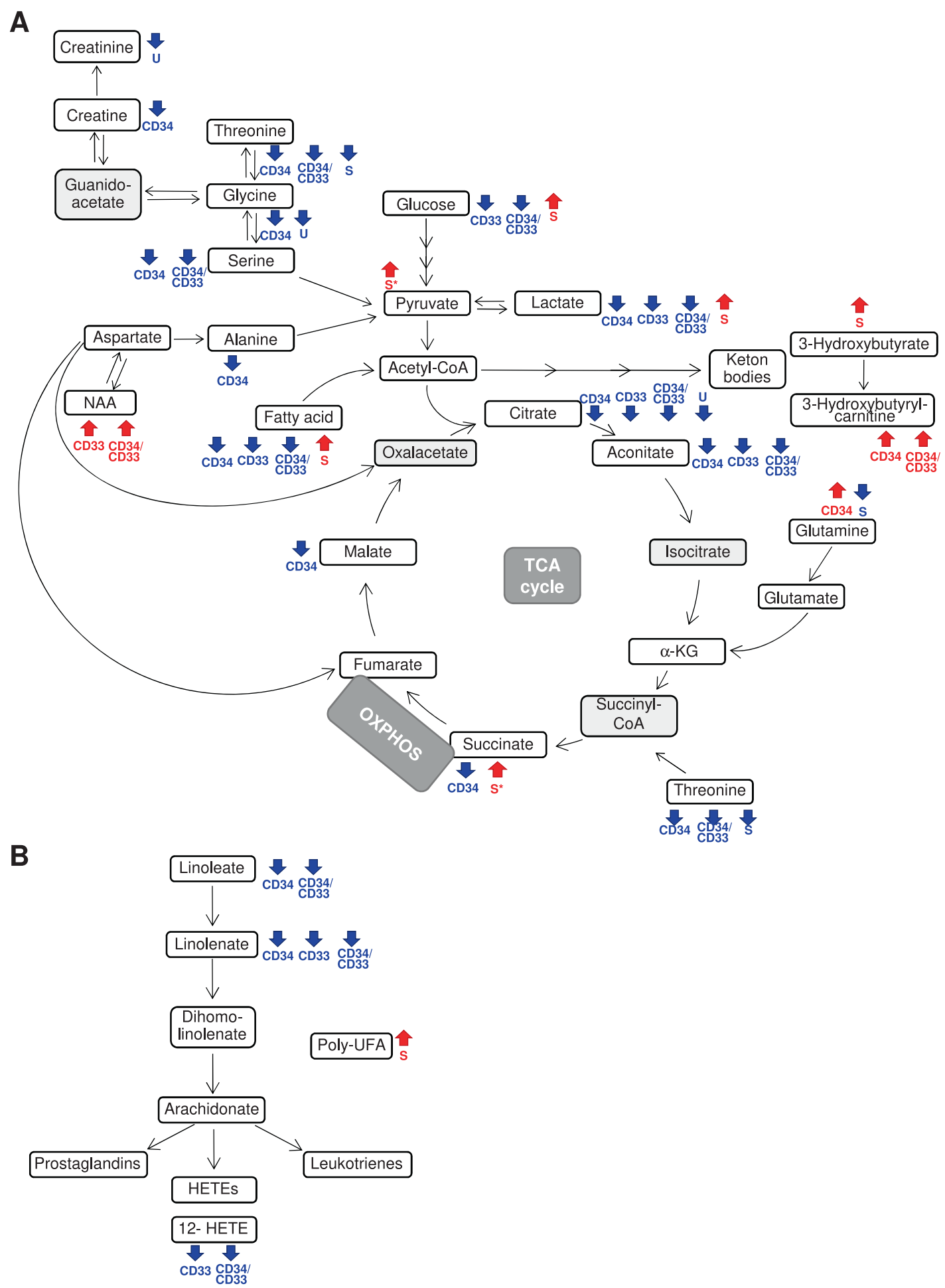

Fig. 4 Summary of AML metabolic alterations in the TCA cycle, linoleic acid metabolism, and related pathways. A TCA cycle and related amino acid pathways. B Linoleic acid metabolism. Red and blue arrows/text indicate increased or decreased metabolite levels in AML cells versus their $C T R L$, respectively (|fold change $\mid \geq 2, q \leq 0.05$ ) and in the serum or urine of patients compared with CTRL $(p<0.05$ ). Gray metabolite boxes indicate the ones that were not detected by MS analysis. The schemes report the most relevant metabolites in the pathway according to metabolomic data (HETE hydroxyeicosatetraenoic acid, NAA N-acetylaspartate, poly-UFA polyunsaturated fatty acids, TCA trycarboxylic acid cycle).

(ADCY9, DPYSL2), lipid (LPL) and carbohydrate (CHST13) metabolism, energy production (CYP1B1) and transporter/exchanger (SLC8A1, SLC1A3, Figs. 7C and S6B). We thus modeled the consequences of gene expression alterations of NPM1/cohesin- mut $\mathrm{AML}$ on the intracellular metabolome by reconstructing genome scale metabolic network models. Based on the analysis of diverse cellular models (Fig. S7A) and our MS data (Fig. S7B), we selected a hematopoietic model derived from Recon2. The 
A

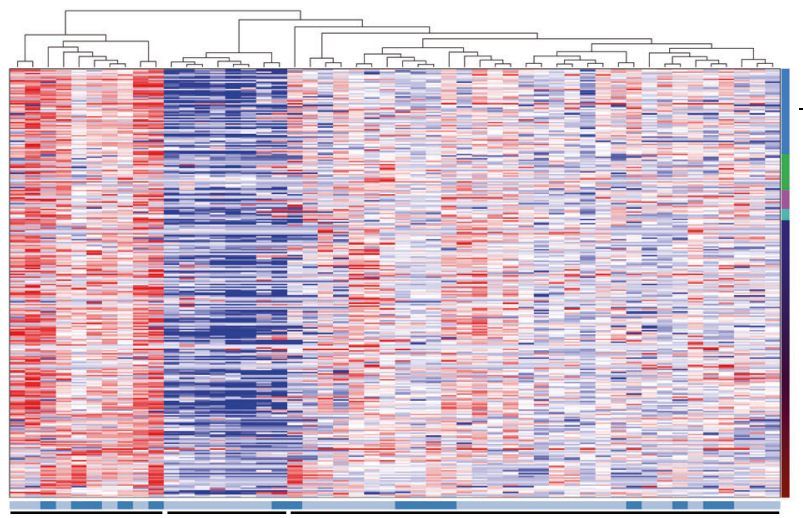

Cluster 1 Cluster 2

Cluster 3

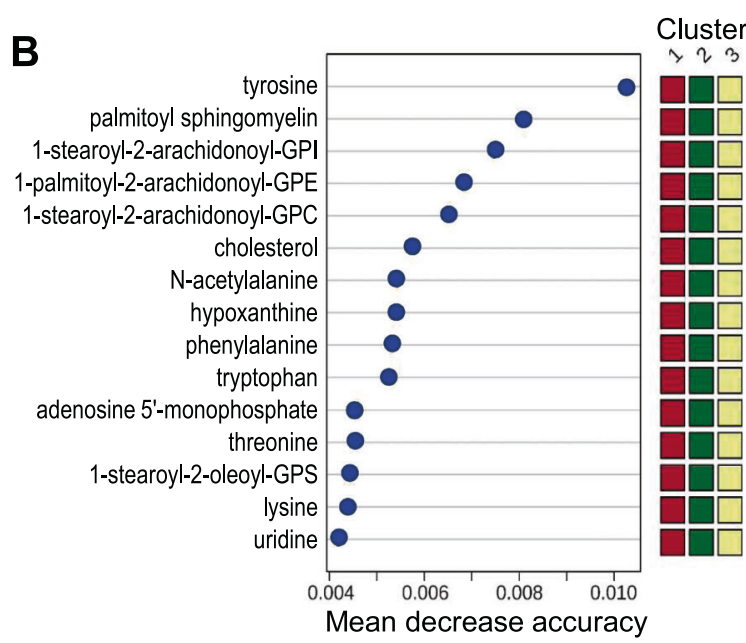

D

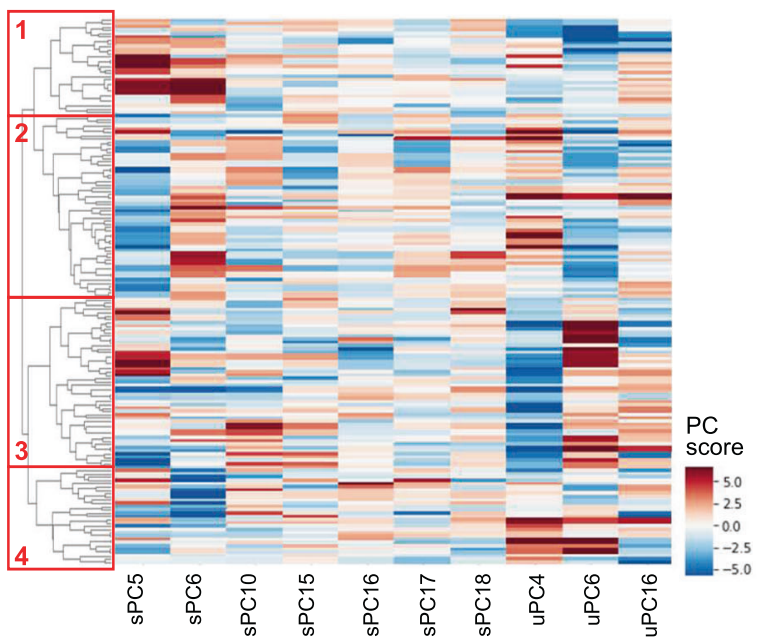

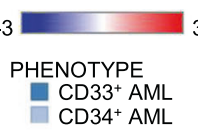

SUPERPATHWAY

Amino Acid
Carbohydrate

Carbohydrate

Energy

Lipid

Nucleotide

Peptide

C

\section{MS cluster 1}

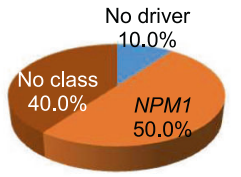

MS cluster 2

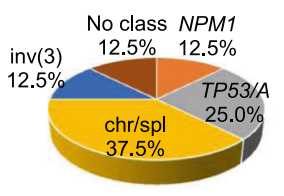

MS cluster 3

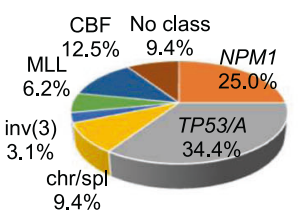

$\mathbf{F}$

E
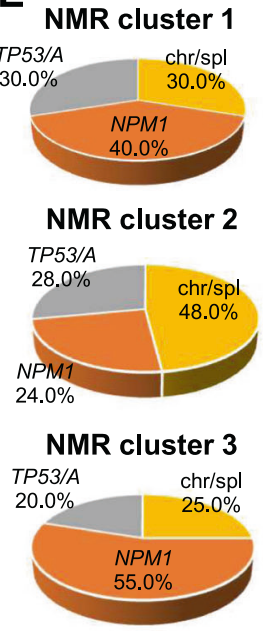

NMR cluster 4

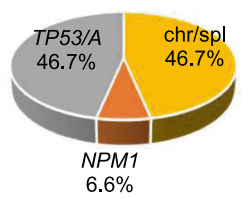

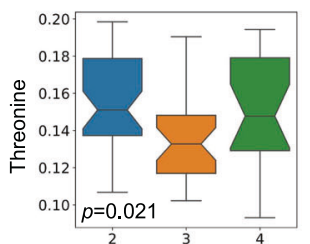
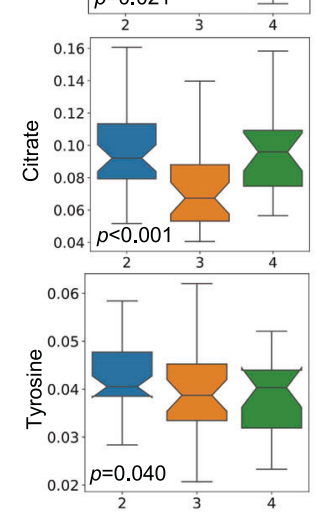

NMR cluster 2

NMR cluster 3

NMR cluster 4 selected reconstruction was validated by modeling the effect of IDH mutations (Table S13 and Fig. S7C, D).

We first predicted the changes in metabolic fluxes (Table S14) induced by the altered expression of enzymes between NPM1-mut and NPM1-wt AML (transcriptomic data from the TCGA and BEAT AML cohorts, Fig. S8). Interestingly, among the perturbed metabolites, experimental evidence confirmed increased $\mathrm{N}$ acetylaspartate and glutamine, reduced spermidine levels (among others) in NPM1-mut compared with NPM1-wt AML (Fig. 7A). We then simulated the intracellular metabolome of NPM1/cohesin- mut AML by adding the 7 downregulated genes to the model (Table S15). Eleven metabolites and 42 reactions were predicted to be specifically perturbed in the NPM1/cohesin-mut model (Table S16). A metabolic network reconstruction of the altered reactions showed a major cluster centered on nicotinate, nicotinamide, and inosine exchange/modification, with connections to glycolysis and metabolism of leukotriene inflammatory molecules (Fig. 7B), that were also confirmed by pathway enrichment analysis of genes catalyzing the network reactions (Fig. S9 and Table S17). Notably, NPM1/cohesin-mut AML showed 
Fig. 5 Intracellular and biofluid metabolomics show association with AML molecular classification. A Unsupervised hierarchical clustering of AML according to intracellular metabolomic profiles (MS, each row denotes a metabolite, each column a sample). B Top 15 metabolites contributing to separation of the three MS metabolic clusters $(1,2,3)$. The metabolites belong to the following superpathways: amino acids and their derivatives (tyrosine, $\mathrm{N}$-acetylalanine, phenilalanine, tryptophan, threonine, lysine), intermediates of purine and pyrimidine metabolism (hypoxanthine, adenosyne-5'-monophosphate, uridine) and lipids (sphingolipid, phosphatidylinositol, phosphatidylethanolamine, phosphatidylcholine, cholesterol, phosphatidylserine. Colored squares on the right indicate metabolite levels in each cluster. C Molecular classification of MS metabolic clusters [3]. Due to the low number of $t(8 ; 21)$ and inv $(16) / t(16 ; 16)$ cases, they were grouped in the core-binding factor (CBF) category and a $\mathrm{t}(6 ; 9)$ patient with complex karyotype was included in the TP53/aneuploidy category (NPM1 NPM1mut, chr/spl chromatin/spliceosome-mut, TP53/A TP53-mut/aneuploidy, inv(3) inv(3)/t(3;3), KMT2A KMT2A-rearranged). D Hierarchical clustering of AML patients belonging to the NPM1-mut, chromatin/spliceosome-mut or TP53/aneuploidy molecular classes according to biofluid metabolomic profile (NMR). These components were selected as the combination of urine and serum spectral features that best described the above mentioned genomic stratification. Of the ten features selected via stochastic gridsearch, seven came from serum spectra, indicating serum as the principal vector of information for this particular stratification. Colors indicate the score on each PC. E Molecular classification of NMR metabolic clusters. F Top scoring serum metabolites separating NMR clusters 2, 3, and 4. Signature metabolites were extracted from sera samples by selecting the highest scoring signals in terms of presence amongst the sera PC responsible for the best separation of molecular subgroups and the average of absolute values of their loadings. Statistical significance was obtained with SciPy.Stats Kruskal-Wallis $H$-test using stepdown Sidak correction. Notch width corresponds to the confidence interval of the median.

lower intracellular levels of inosine-5'-monophosphate and glucose when compared with NPM1-wt and/or NPM1-mut AML (Fig. 7C). Overall, our multistep approach defined the metabolic specificities of $N P M 1 /$ cohesin-mut AML.

\section{DISCUSSION}

Few studies have previously analyzed the metabolic profile of AML patient serum [37-41] or of a limited number of primary cells $[17,42,43]$. Here, we have performed integrated genomics and metabolomics analysis in AML, which showed genetic-related differences in the metabolic profiles and defined multiple subgroups with distinct constellations of mutations and metabolic features.

Among the endogenous factors influencing the human metabolome, age and sex have a strong influence, at least in healthy subjects [44]. However, we can exclude confounding effects in our results for the following reasons: (i) the majority of metabolic alterations occurred in the opposite direction to the one expected as a readout of confounding factors [44]; (ii) the correlation coefficient for the identified metabolites with age and gender was not significant; (iii) some of the data obtained on serum were also reported in previous studies [37-41].

First, integrated serum and urine analysis accurately discriminated between AML and normal patients, suggesting a robust approach for evaluating disease metabolic subgroups and a valid, low-cost approach for noninvasive population analyses.

Second, we integrated biofluid and intracellular metabolomics. We used NMR and MS as complementary techniques for biofluids and primary cell profiling, respectively. The rationale of this approach is twofold: it allowed us to benefit from the reproducibility of NMR, which offers unbiased information and could enable a rapid translation to the clinical practice, and from the high sensitivity of MS in metabolite detection from low cell numbers. Our comprehensive view showed alterations in the TCA cycle and in the metabolism of purine nucleotides, amino acid, fatty acids, keton bodies, polyamine, glutamine and other amino acids. The incomplete overlap between the metabolic alterations observed when comparing $\mathrm{CD} 34^{+}$and $\mathrm{CD}_{3} 3^{+}$blasts with their respective healthy populations may be partly due to the usage of CD34 ${ }^{+} \mathrm{CB}$ and $\mathrm{CD}_{3}{ }^{+} \mathrm{PB}$ cells, as controls. Notably, many of the identified pathways can be therapeutically exploited (e.g. glutaminolysis, arginine uptake, aspartate production, fatty acid oxidation, polyamine metabolism, ketogenesis) and the inhibition of some of them achieved promising results in $A M L[17,45]$ or in cancer $[46,47]$ models.

When integrated with genetic features, the metabolic profiles showed association with NPM1-mut, chromatin/spliceosome-mut and TP53-mut/aneuploid AML classes. Chromatin/spliceosome- mut and TP53-mut/aneuploid AML shared some metabolic features, according to their clustering. This may be partly explained by the recurrence of aneuploidies in the chromatin/ spliceosome-mut class (42.9\% of those classified in the TP53-mut/ aneuploid AML associated cluster). NPM1-mut AML showed high intracellular levels of $\mathrm{N}$-acetyl-aspartyl-glutamate, that have been previously associated with MYC activation [48]. Indeed, mutant NPM1 indirectly stabilizes c-MYC protein [49] and an oncogenic MYC mutation was also detected in a NPM1-mut AML case, in line with previous findings [3].

Our data also classified NPM1-mut AML carrying mutations in cohesin or DNA damage-related genes as a distinct metabolic subgroup. This group does not associate with IDH1-2/TET2 mutations, which are also frequently observed in NPM1-mut cases $[1,2,50]$ but it was characterized by higher mutation burden, lower white blood cell count and dowregulation of immunerelated genes [51]. Accordingly, in silico modeling of the NPM1/ cohesin-mut-specific metabolic perturbations predicted changes in the balance of leukotrienes. Among the metabolic genes downregulated in NPM1/cohesin-mut compared with NPM1-mut AML, CHST13, ADCY9, PRR16, LPL, and SLC1A3 were confirmed by STAG2-deficient model of NPM1-mut leukemia [52]. Moreover, flux and network analysis based on the identified transcriptomic changes pointed at alterations in the purine and NAD superpathways as NPM1/cohesin-mut-specific ones. Inosine-5'-monosphate, an intermediate in the purine metabolism, showed low levels in NPM1/cohesin-mut compared with NPM1-mut AML cells. Of note, inosine favors spontaneous mutations, since a base transition is introduced when it is incorporated into newly synthesized DNA [53]. In addition, both purines and NAD regulate immune function and cytokine release $[54,55]$. Given that NAD modulates gene expression [56] and that cohesin gene mutations alter chromatin accessibility [57], the targeting of NAD metabolism could restore the myeloid differentiation program in NPM1/ cohesin-mut leukemic cells. Future studies taking into account leukemic cell metabolism and microenvironmental factors, will further investigate the suggested vulnerabilities. With regard to therapeutics, NPM1/cohesin-mut AML were more sensitive than NPM1-mut AML to EGFR inhibition, which may lead to the release of the differentiation brake [58] and to drugs targeting the tyrosine kinase receptor MET, likely due to a mild autocrine pathway activation in these cases, who express low levels of the ligand [59].

Overall, our results provide a map of the crosstalk between metabolic pathways and between genomics and metabolomics in $A M L$, reflecting functional interactions and dependencies that could be therapeutically exploited and provide the rationale for a switch to a genomic- and phenotypic-driven personalized medicine. 
A
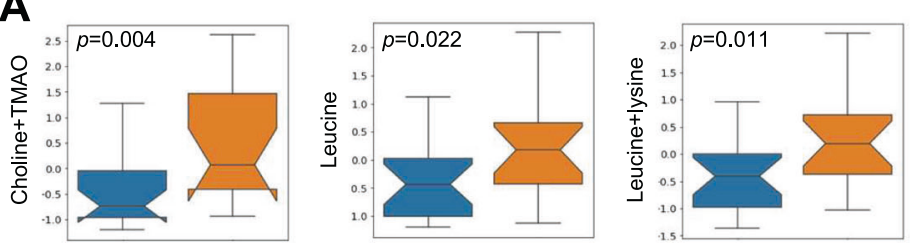

NMR NPM1-mut NMR NPM1/cohesin-mut

\section{B}

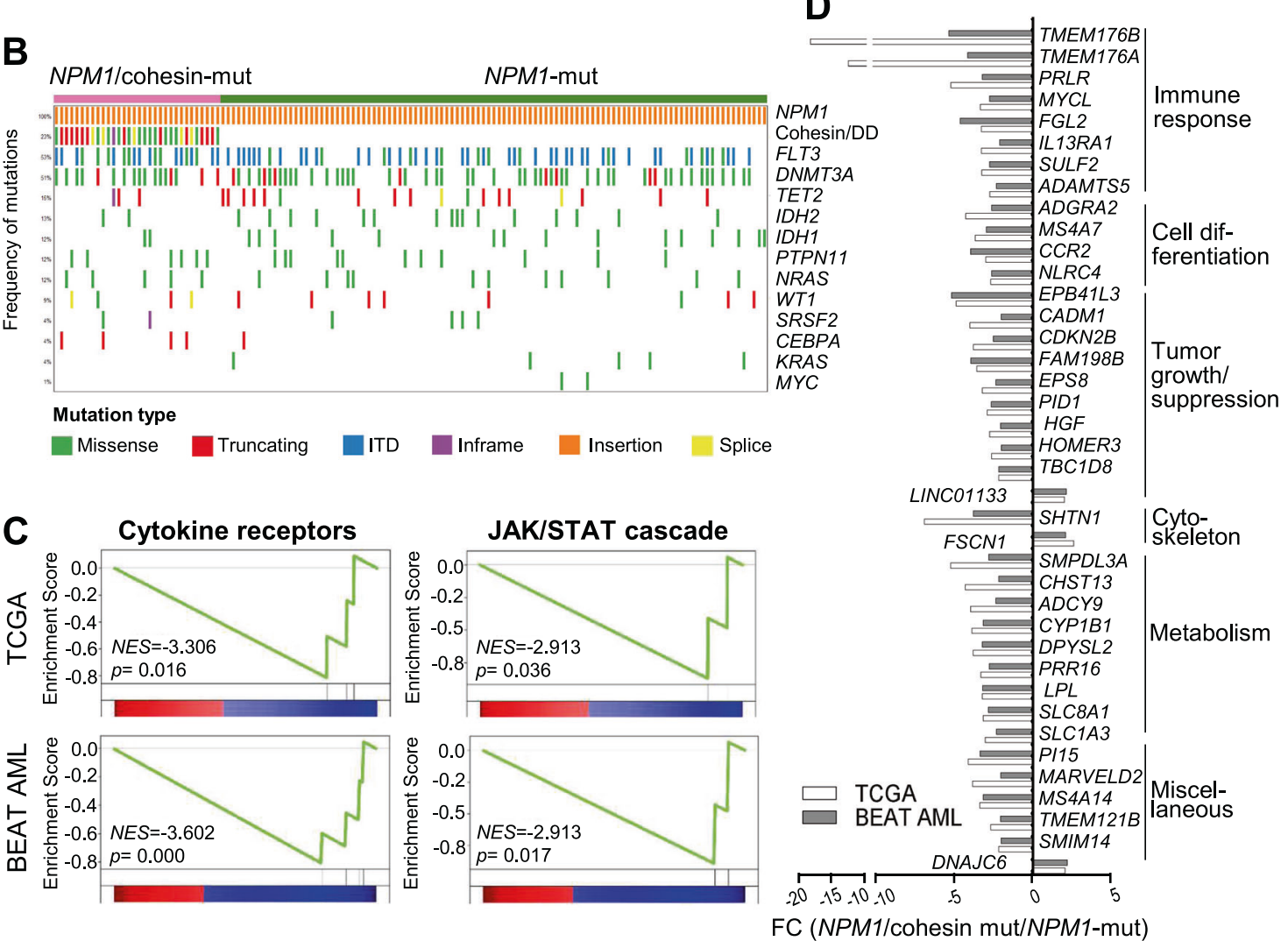

D

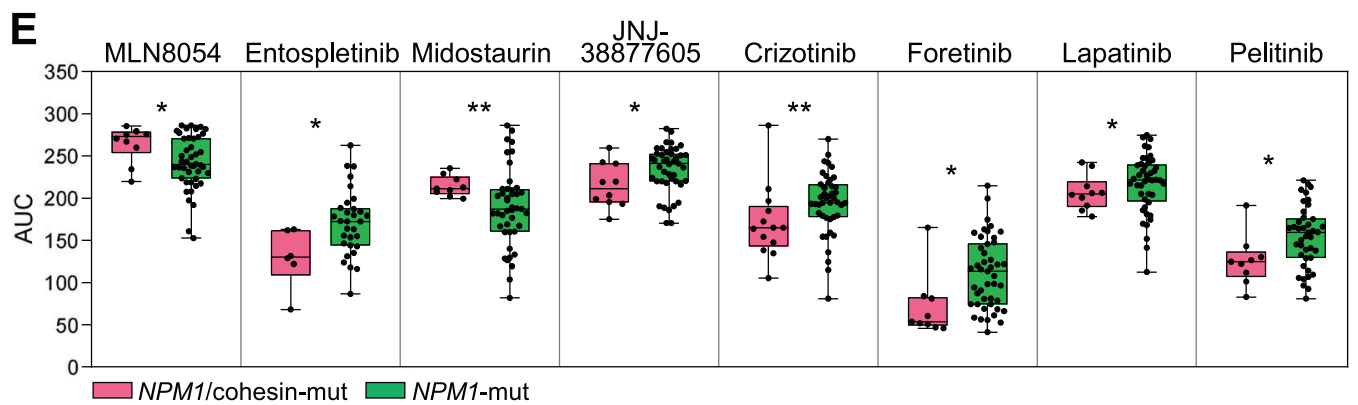

Fig. 6 Metabolic, genomic, transcriptomic and drug response differences between NPM1/cohesin-mut and NPM1-mut AML. A Serum metabolites separating NPM1/cohesin-mut and NPM1-mut AML (TMAO trimethylamine-N-oxide). B Oncoprint of mutations in AML-related genes (frequency $>3 \%$ in the overall population) in NPM1/cohesin-mut and NPM1-mut AML. WES data were obtained from the TCGA ( $n=13$ NPM1/cohesin-mut, $n=33$ NPM1-mut) and BEAT AML ( $n=19$ NPM1/cohesin-mut, $n=72$ NPM1-mut, including 7 relapse cases) cohorts. Rows denote genes or groups of genes (cohesin/DD cohesion/DNA damage-related genes). Columns represent frequency of mutations and single patients (ITD internal tandem duplication). C Signatures of cytokine receptors and JAK-STAT cascade from GSEA showing significance in both datasets (TCGA, left to right: cytokine-cytokine receptor binding, regulation of JAK-STAT cascade, $n=9$ NPM1/cohesin-mut, $n=25$ NPM1-mut; BEAT AML, left to right: cytokine receptor activity, JAK/STAT cascade, $n=14$ NPM1/cohesin-mut, $n=47$ NPM1-mut, including 3 relapse cases). D Genes involved in immune response, cell differentiation, tumor growth regulation, cytoskeleton, metabolism and other cellular processes, showing a significantly different expression between NPM1/cohesin-mut and NPM1-mut AML in both cohorts. E Area under the curve (AUC) for the drugs showing a significantly different response between NPM1/cohesin-mut and NPM1-mut AML was plotted for the two cohorts (NPM1/cohesin-mut, $n=6-13$; NPM1-mut, $n=31-45$ ) [1]: MLN8054 (Aurora kinase A inhibitor), Entospletinib (SYK inhibitor), Midostaurin (FLT3, JAK inhibitor), JNJ-38877605 (MET inhibitor), Crizotinib (ALK, MET, ROS1, NTRK inhibitor), Foretinib (MET, KDR, TIE inhibitor), Lapatinib (ErbB-2, EGFR inhibitor), Pelitinib (EGFR inhibitor). Boxes represent the mean (horizontal line) and extend from the $25^{\text {th }}$ to $75^{\text {th }}$ percentiles; whiskers extend from the minimum to the maximum value and each value is plotted $\left({ }^{*} p \leq 0.05,{ }^{* *} p \leq 0.01\right)$. 
A

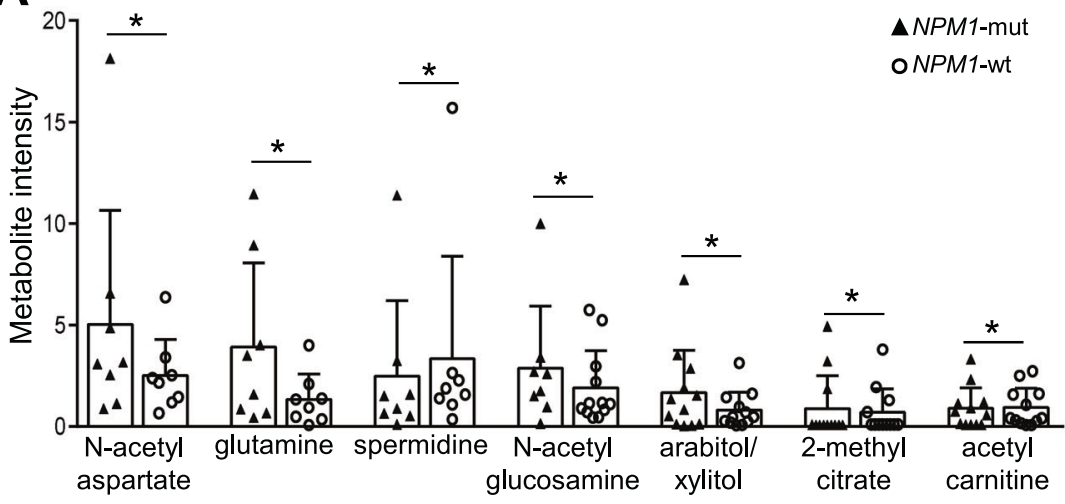

C

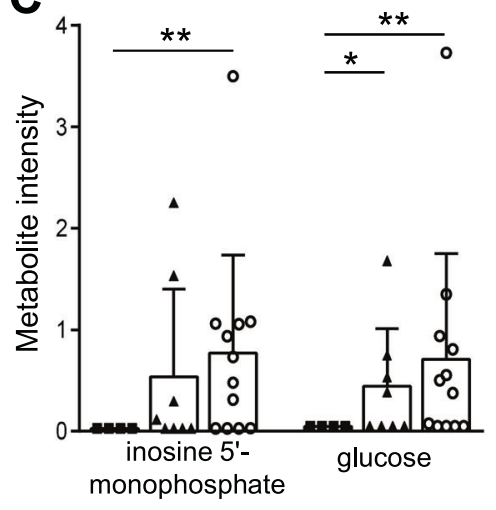

NPM1-double-mut

$\triangle N P M 1-$ mut

o NPM1-wt

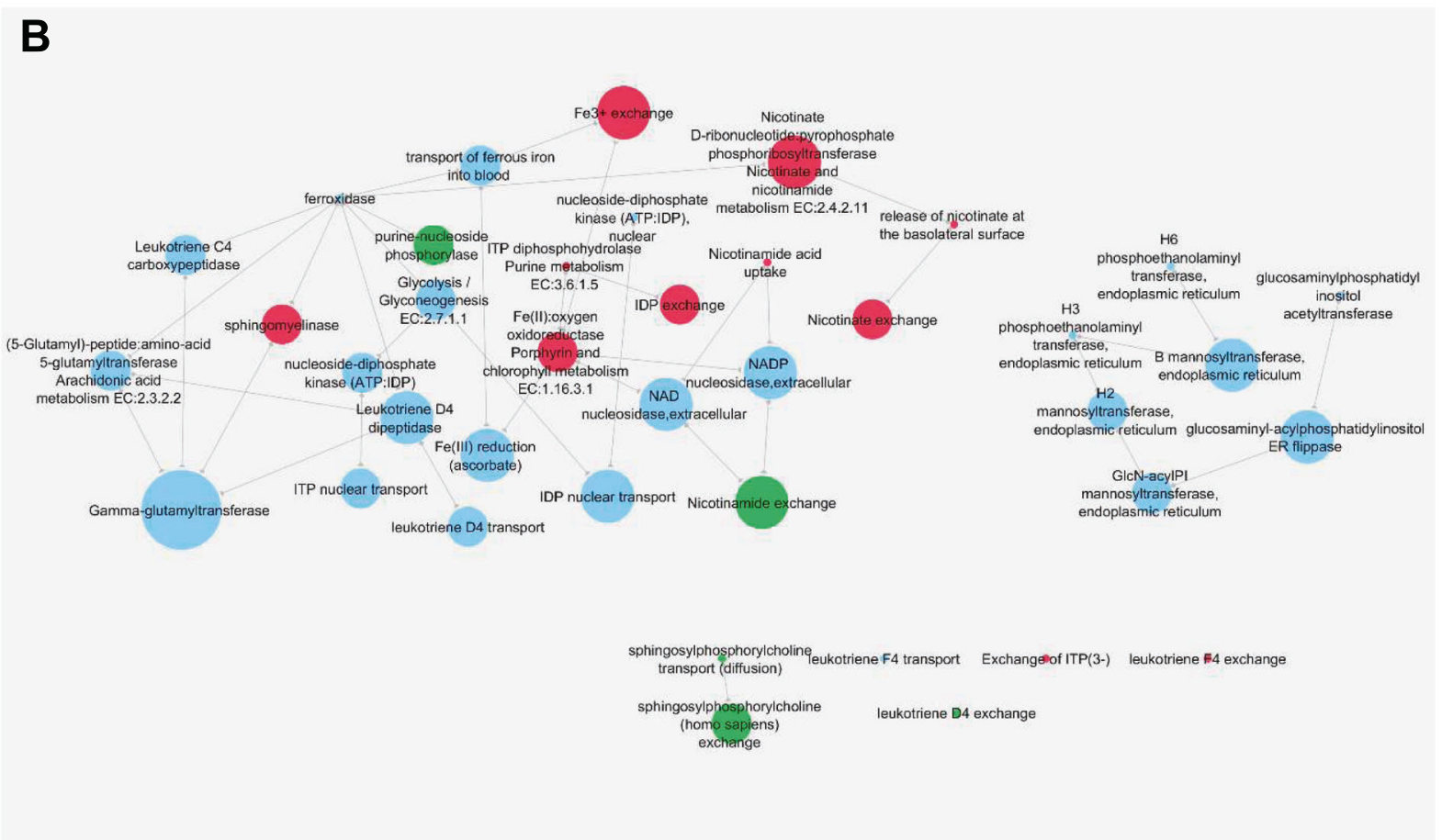

Fig. 7 Modeling the metabolic network of NPM1/cohesin-mut AML. A Intracellular metabolites from FVA analysis showing a significant different intensity between NPM1-mut $(n=8)$ and NPM1-wt $(n=12$, normal karyotype) in MS analysis. Metabolite intensity normalized on DNA concentration is shown in the plot. Significance was obtained by Welch $t$-test $\left({ }^{*} p \leq 0.05\right)$. B NPM1/cohesin-mut specific metabolic reaction perturbation network. (red: minimum flux, green: maximum flux, light blue: no information among NPM1/cohesin-mut-specific alterations). Sizes of nodes are proportional to links originating from that node and pointing towards others (outdegree). C Intracellular metabolite intensity of NPM1/cohesin-mut-specific perturbations predicted by FVA (NPM1/cohesin-mut, $n=4 ; N P M 1$-mut, $n=8, N P M 1$-wt, $n=12$, normal karyotype). Metabolite intensity normalized on DNA concentration is shown in the plot. Significance was obtained by Welch $t$-test $\left({ }^{*} p \leq 0.05\right.$, $\left.*^{* *} p \leq 0.01\right)$.

\section{REFERENCES}

1. Tyner JW, Tognon CE, Bottomly D, Wilmot B, Kurtz SE, Savage SL, et al. Functional genomic landscape of acute myeloid leukaemia. Nature. 2018;562:526-31.

2. TCGA TCGA. Genomic and epigenomic landscapes of adult de novo acute myeloid leukemia. N. Engl J Med. 2013;368:2059-74.

3. Papaemmanuil E, Gerstung M, Bullinger L, Gaidzik VI, Paschka P, Roberts ND, et al. Genomic classification and prognosis in acute myeloid leukemia. N. Engl J Med. 2016;374:2209-21.

4. Tzoulaki I, Castagné R, Boulangé $C L$, Karaman I, Chekmeneva E, Evangelou E, et al. Serum metabolic signatures of coronary and carotid atherosclerosis and subsequent cardiovascular disease. Eur Heart J. 2019;40:2883-96.
5. Barbara G, Scaioli E, Barbaro MR, Biagi E, Laghi L, Cremon C, et al. Gut microbiota, metabolome and immune signatures in patients with uncomplicated diverticular disease. Gut. 2017;66:1252-61.

6. Liu J, Semiz S, van der Lee SJ, van der Spek A, Verhoeven A, van Klinken JB, et al. Metabolomics based markers predict type 2 diabetes in a 14-year follow-up study. Metabolomics. 2017;13:104.

7. Hasim A, Ali M, Mamtimin B, Ma JQ, Li QZ, Abudula A. Metabonomic signature analysis of cervical carcinoma and precancerous lesions in women by $1 \mathrm{H}$ NMR spectroscopy. Exp Ther Med. 2012;3:945-51.

8. Puchades-Carrasco L, Pineda- Lucena A. Metabolomics applications in precision medicine: an oncological perspective. Curr Top Med Chem. 2017;17:2740-51. 
9. Ward PS, Patel J, Wise DR, Abdel-Wahab O, Bennett BD, Coller HA, et al. The common feature of leukemia-associated IDH1 and IDH2 mutations is a neomorphic enzyme activity converting a-ketoglutarate to 2-hydroxyglutarate. Cancer Cell. 2010;17:225-34.

10. Fathi AT, Sadrzadeh H, Borger DR, Ballen KK, Amrein PC, Attar EC, et al. Prospective serial evaluation of 2-hydroxyglutarate, during treatment of newly diagnosed acute myeloid leukemia, to assess disease activity and therapeutic response. Blood. 2012;120:4649-52.

11. Figueroa ME, Abdel-Wahab O, Lu C, Ward PS, Patel J, Shih A, et al. Leukemic IDH1 and IDH2 mutations result in a hypermethylation phenotype, disrupt TET2 function, and impair hematopoietic differentiation. Cancer Cell. 2010;18:553-67.

12. Chaturvedi A, Araujo Cruz MM, Jyotsana N, Sharma A, Goparaju R, Schwarzer A, et al. Enantiomer-specific and paracrine leukemogenicity of mutant IDH metabolite 2-hydroxyglutarate. Leukemia. 2016;30:1708-15.

13. DiNardo CD, Propert KJ, Loren AW, Paietta E, Sun Z, Levine RL, et al. Serum 2hydroxyglutarate levels predict isocitrate dehydrogenase mutations and clinical outcome in acute myeloid leukemia. Blood. 2013;121:4917-24.

14. Ye H, Adane B, Khan N, Alexeev E, Nusbacher N, Minhajuddin M, et al. Subversion of systemic glucose metabolism as a mechanism to support the growth of leukemia cells. Cancer Cell. 2018;34:659-.e6.

15. Škrtić M, Sriskanthadevan S, Jhas B, Gebbia M, Wang X, Wang Z, et al. Inhibition of mitochondrial translation as a therapeutic strategy for human acute myeloid leukemia. Cancer Cell. 2011;20:674-88.

16. Lagadinou ED, Sach A, Callahan K, Rossi RM, Neering SJ, Minhajuddin M, et al. $\mathrm{BCL}-2$ inhibition targets oxidative phosphorylation and selectively eradicates quiescent human leukemia stem cells. Cell Stem Cell. 2013;12:329-41.

17. Jones $C L$, Stevens BM, D'Alessandro A, Reisz JA, Culp-Hill R, Nemkov T, et al. Inhibition of amino acid metabolism selectively targets human leukemia stem cells. Cancer Cell. 2018;34:724-.e4.

18. Jones CL, Stevens BM, D'Alessandro A, Culp-Hill R, Reisz JA, Pei S, et al. Cysteine depletion targets leukemia stem cells through inhibition of electron transport complex II. Blood. 2019;134:389-94.

19. Jacque N, Ronchetti AM, Larrue C, Meunier G, Birsen R, Willems L, et al. Targeting glutaminolysis has antileukemic activity in acute myeloid leukemia and synergizes with BCL-2 inhibition. Blood. 2015;126:1346-56.

20. Molina JR, Sun Y, Protopopova M, Gera S, Bandi M, Bristow C, et al. An inhibitor of oxidative phosphorylation exploits cancer vulnerability. Nat Med. 2018;24:1036-46.

21. Mussai F, Egan S, Higginbotham-Jones J, Perry T, Beggs A, Odintsova E, et al. Arginine dependence of acute myeloid leukemia blast proliferation: a novel therapeutic target. Blood. 2015;125:2386-96.

22. Gallipoli P, Giotopoulos G, Tzelepis K, Costa ASH, Vohra S, Medina-Perez P, et al. Glutaminolysis is a metabolic dependency in FLT3 ITD acute myeloid leukemia unmasked by FLT3 tyrosine kinase inhibition. Blood. 2018;131:1639-53.

23. Fenouille N, Bassil CF, Ben-Sahra I, Benajiba L, Alexe G, Ramos A, et al. The creatine kinase pathway is a metabolic vulnerability in EVI1-positive acute myeloid leukemia. Nat Med. 2017;23:301-13.

24. Barve Vega, Shah Ghare, Casson Wunderlich, et al. Perturbation of methionine/Sadenosylmethionine metabolism as a novel vulnerability in MLL rearranged leukemia. Cells. 2019;8:1322.

25. Ju HQ, Zhan G, Huang A, Sun Y, Wen S, Yang J, et al. ITD mutation in FLT3 tyrosine kinase promotes Warburg effect and renders therapeutic sensitivity to glycolytic inhibition. Leukemia. 2017;31:2143-50.

26. Simonetti G, Padella A, do Valle IF, Fontana MC, Fonzi E, Bruno S, et al. Aneuploid acute myeloid leukemia exhibits a signature of genomic alterations in the cell cycle and protein degradation machinery. Cancer. 2018;125:712-25.

27. Shlomi T, Cabili MN, Ruppin E. Predicting metabolic biomarkers of human inborn errors of metabolism. Mol Syst Biol. 2009;5:263.

28. van Rossum G, Drake FL. Python 3 Reference Manual. 2009.

29. Virtanen P, Gommers R, Oliphant TE, Haberland M, Reddy T, Cournapeau D, et al. SciPy 1.0: fundamental algorithms for scientific computing in Python. Nat Methods. 2020;17:261-72.

30. Seabold S, Perktold J Statsmodels: Econometric and Statistical Modeling with Python. In: Proceedings of the 9th Python in Science Conference. 2010. https:// doi.org/10.25080/majora-92bf1922-011.

31. R Core Team (2020). R: A language and environment for statistical computing. R A Lang. Environ. Stat. Comput. R Found. Stat. Comput. Vienna, Austria. 2020. https:// www.r-project.org/.

32. Itahana Y, Itahana K. Emerging roles of p53 family members in glucose metabolism. Int J Mol Sci. 2018;19:776.

33. Boidot R, Veǵran F, Meulle A, Le Breton A, Dessy C, Sonveaux P, et al. Regulation of monocarboxylate transporter MCT1 expression by p53 mediates inward and outward lactate fluxes in tumors. Cancer Res. 2012;72:939-48.
34. Pegg AE. Polyamine metabolism and its importance in neoplastic growth and as a target for chemotherapy. Cancer Res. Cancer Res. 1988;48:759-74.

35. Sumita K, Lo YH, Takeuchi K, Senda M, Kofuji S, Ikeda Y, et al. The lipid kinase PI5P4K $\beta$ is an intracellular GTP sensor for metabolism and tumorigenesis. Mol Cell. 2016;61:187-98.

36. Zand B, Previs RA, Zacharias NM, Rupaimoole R, Mitamura T, Nagaraja AS, et al. Role of Increased n-acetylaspartate Levels in Cancer. J Natl Cancer Inst. 2016;108: djv426.

37. Musharraf SG, Siddiqui AJ, Shamsi T, Naz A. SERUM metabolomics of acute lymphoblastic leukaemia and acute myeloid leukaemia for probing biomarker molecules. Hematol Oncol. 2017:35:769-77.

38. Grønningsæter IS, Fredly HK, Gjertsen BT, Hatfield KJ, Bruserud $\varnothing$. Systemic metabolomic profiling of acute myeloid leukemia patients before and during disease-stabilizing treatment based on all-trans retinoic acid, valproic acid, and low-dose chemotherapy. Cells. 2019;8:1229.

39. Chen WL, Wang JH, Zhao AH, Xu X, Wang $\mathrm{YH}$, Chen $\mathrm{TL}$, et al. A distinct glucose metabolism signature of acute myeloid leukemia with prognostic value. Blood. 2014;124:1645-54.

40. Wang Y, Zhang L, Chen WL, Wang JH, Li N, Li JM, et al. Rapid diagnosis and prognosis of de novo acute myeloid leukemia by serum metabonomic analysis. J Proteome Res. 2013;12:4393-401.

41. Wojtowicz W, Chachaj A, Olczak A, Ząbek A, Piątkowska E, Rybka J, et al. Serum NMR metabolomics to differentiate haematologic malignancies. Oncotarget. 2018;9:24414-27.

42. Bhanot $H$, Reddy MM, Nonami A, Weisberg EL, Bonal D, Kirschmeier PT, et al. Pathological glycogenesis through glycogen synthase 1 and suppression of excessive AMP kinase activity in myeloid leukemia cells. Leukemia. 2015;29:1555-63.

43. Stockard B, Garrett T, Guingab-Cagmat J, Meshinchi S, Lamba J. Distinct metabolic features differentiating FLT3-ITD AML from FLT3-WT childhood acute myeloid leukemia. Sci Rep. 2018;8:5534.

44. Rist MJ, Roth A, Frommherz L, Weinert $\mathrm{CH}$, Krüger R, Merz B, et al. Metabolite patterns predicting sex and age in participants of the Karlsruhe Metabolomics and Nutrition (KarMeN) study. PLoS One. 2017;12:e0183228.

45. Pollyea DA, Stevens $B M$, Jones $C L$, Winters $A$, Pei $S$, Minhajuddin $M$, et al. Venetoclax with azacitidine disrupts energy metabolism and targets leukemia stem cells in patients with acute myeloid leukemia. Nat Med. 2018;24:1859-66.

46. Puchalska P, Crawford PA. Multi-dimensional roles of ketone bodies in fuel metabolism, signaling, and therapeutics. Cell Metab. 2017;25:262-84.

47. Casero RA, Murray Stewart T, Pegg AE. Polyamine metabolism and cancer: treatments, challenges and opportunities. Nat Rev Cancer. 2018;18:681-95.

48. Nguyen T, Kirsch BJ, Asaka R, Nabi K, Quinones A, Tan J, et al. Uncovering the role of $\mathrm{N}$-acetyl-aspartyl-glutamate as a glutamate reservoir in cancer. Cell Rep. 2019;27:491-501.e6.

49. Bonetti P, Davoli T, Sironi C, Amati B, Pelicci PG, Colombo E. Nucleophosmin and its AML-associated mutant regulate c-Myc turnover through Fbw7 $\gamma$. J Cell Biol. 2008;182:19-26.

50. Mason EF, Kuo FC, Hasserjian RP, Seegmiller AC, Pozdnyakova O. A distinct immunophenotype identifies a subset of NPM1-mutated AML with TET2 or IDH1/ 2 mutations and improved outcome. Am J Hematol. 2018;93:504-10.

51. Cuartero S, Weiss FD, Dharmalingam G, Guo Y, Ing-Simmons E, Masella S, et al. Control of inducible gene expression links cohesin to hematopoietic progenitor self-renewal and differentiation. Nat Immunol. 2018;19:932-41.

52. Smith JS, Lappin KM, Craig SG, Liberante FG, Crean CM, McDade SS, et al. Chronic loss of STAG2 leads to altered chromatin structure contributing to de-regulated transcription in AML. J Transl Med. 2020;18:339.

53. Alseth I, Dalhus B, Bjørås M. Inosine in DNA and RNA. Curr Opin Genet Dev. 2014:26:116-23

54. Minhas PS, Liu L, Moon PK, Joshi AU, Dove C, Mhatre S, et al. Macrophage de novo NAD+ synthesis specifies immune function in aging and inflammation. Nat Immunol. 2019:20:50-63.

55. Antonioli L, Blandizzi C, Pacher P, Haskó G. Immunity, inflammation and cancer: a leading role for adenosine. Nat Rev Cancer. 2013;13:842-57.

56. Yaku K, Okabe K, Hikosaka K, Nakagawa T. NAD metabolism in cancer ther apeutics. Front Oncol. 2018;8:622.

57. Viny AD, Levine RL. Cohesin mutations in myeloid malignancies made simple. Curr Opin Hematol. 2018;25:61-6.

58. Stegmaier K, Corsello SM, Ross KN, Wong JS, DeAngelo DJ, Golub TR. Gefitinib induces myeloid differentiation of acute myeloid leukemia. Blood. 2005;106:2841-8.

59. Kentsis A, Reed C, Rice KL, Sanda T, Rodig SJ, Tholouli E, et al. Autocrine activation of the MET receptor tyrosine kinase in acute myeloid leukemia. Nat Med. 2012;18:1118-22. 


\section{ACKNOWLEDGEMENTS}

We thank all of our patients and healthy volunteers for donating precious time and tissue. We thank Sante Tura, Maria Lia Lunardelli, Annarita Belardinelli and Associazione Italiana contro le Leucemie, linfomi e mielomi (AIL) Bologna for their help in coordinating the study; Grainne Eileen Tierney for editorial assistance. Funding for this project was provided, in part, by an EHA Research Fellowship award granted by the European Hematology Association (to GS). The study was also supported by grants from the Seventh Framework Programme (FP7/2007-2013, GA n '306242-NGS-PTL), Italian Ministry of Health RRC-2020-23670007 (ERAPERMED2019290) and GR-2018-12365278, Torsten Haferlach Leukämiediagnostik Stiftung, Fondazione del Monte di Bologna e Ravenna (ID ROL: FdM/4007).

\section{COMPETING INTERESTS}

The authors declare the following competing interests: II received honoraria from Amgen; TH is part owner of MLL Munich Leukemia Laboratory; GM reports compensation from Amgen, Incyte, Janssen, Jazz Pharmaceuticals, AbbVie and Daiichi Sankyo (consultancy), Pfizer and Celgene (consultancy and speakers' bureau), Novartis (speakers' bureau), outside the submitted work. The other authors have declared that no conflict of interest exists.

\section{INFORMED CONSENT}

The study was approved by the Institutional Ethics Committees (protocol 253/2013/ O/Tess, 112/2014/U/Tess, Policlinico Sant'Orsola-Malpighi; internal MLL board and SOP-EN-ISO-15189) and was carried out in accordance with the principles laid down in the 1964 Declaration of Helsinki. Written informed consent was received from participants prior to inclusion in the study.

\section{ADDITIONAL INFORMATION}

Supplementary information The online version contains supplementary material available at https://doi.org/10.1038/s41375-021-01318-x.

Correspondence and requests for materials should be addressed to G.S. or A.P.

Reprints and permission information is available at http://www.nature.com/ reprints

Publisher's note Springer Nature remains neutral with regard to jurisdictional claims in published maps and institutional affiliations.

(i) Open Access This article is licensed under a Creative Commons Attribution 4.0 International License, which permits use, sharing, adaptation, distribution and reproduction in any medium or format, as long as you give appropriate credit to the original author(s) and the source, provide a link to the Creative Commons license, and indicate if changes were made. The images or other third party material in this article are included in the article's Creative Commons license, unless indicated otherwise in a credit line to the material. If material is not included in the article's Creative Commons license and your intended use is not permitted by statutory regulation or exceeds the permitted use, you will need to obtain permission directly from the copyright holder. To view a copy of this license, visit http://creativecommons. org/licenses/by/4.0/.

(c) The Author(s) 2021 